\title{
Ear Drops Emulsion Dosage Form
}

National Cancer Institute

\section{Source}

National Cancer Institute. Ear Drops Emulsion Dosage Form. NCI Thesaurus. Code C149450.

Liquid single-dose or multidose preparation consisting of an emulsion intended for application to the external auditory meatus. Multidose containers may be dropper containers or containers provided with a dropper applicator, or the dropper may be supplied separately. Drops are not necessarily administered dropwise, but may also be administered as a small volume. 